\title{
Properties of fast solitary structures
}

\author{
R. E. Ergun, C. W. Carlson, L. Muschietti, I. Roth, and J. P. McFadden . \\ Space Sciences Laboratory, University of California. Berkeley, CA, 94720, USA
}

Received: 15 June 1999 - Revised: 6 September 1999 - Accepted: 13 September 1999

\begin{abstract}
We present detailed observations of electromagnetic waves and particle distributions from the Fast Auroral SnapshoT (FAST) satellite which reveal many important properties of large-amplitude, spatially-coherent plasma structures known as "fast solitary structures" or "electron phase space holes". Similar structures have been observed in several regions of the magnetosphere including the auroral zone, plasma sheet boundary layer, and bow shock. There has been rapid theoretical progress in understanding these structures. Solitary structures can develop from bidirectional electron beams. Once developed, the one-dimensional properties parallel to the magnetic field can be adequately described by analytical treatment as BGK structures. There remains, however, several unanswered questions. The origin of the bidirectional electron beams, the development of twoor three-dimensional structures, and the observed association with the ion cyclotron frequency are not well understood.
\end{abstract}

\section{Introduction}

In this article, we summarize FAST satellite observations of solitary structures that are associated with energetic electron fluxes in the auroral zone (Ergun et al., 1998a; 1998b; 1998c; Ergun, 1999a). Similar structures have been observed by other auroral spacecraft (Dubouloz et al., 1991; Mozer et al., 1997) and in space plasmas outside of the aurora (Matsumoto et al., 1994; Bale et al., 1998). Fast solitary structures have speeds far greater than the ion thermal speed $\left(v_{i t h}\right)$ and thus were interpreted (Omura et al., 1994) to be one-dimensional electron phase space holes (Saeki et al., 1979; Schamel, 1982; Turikov, 1984).

In the mid-altitude $(\sim 4000 \mathrm{~km})$ auroral zone, these structures travel roughly at the electron drift velocity, have durations of $\sim 100 \mu \mathrm{s}$, and have amplitudes as high as $2.5 \mathrm{~V} / \mathrm{m}$ which distinguishes them from previous observations of ionacoustic solitary waves or weak double layers (Temerin et al., 1982). They appear in power-frequency-time spectrograms as brief, but intense emissions of broadband, quasielectrostatic noise and have been observed at all local times that FAST has covered. They appear to be in or near regions of parallel electric fields (Ergun et al., 1998a; 1998b). Fast solitary structures are relatively common in the downward current region of the aurora but also have been observed, albeit less frequently, in the upward current region. They are only observed in conjunction with intense, field-aligned electron fluxes.

The auroral solitary structures were shown to be Debyescale, positively-charged, three-dimensional clouds surrounded by a negative halo (Ergun et al., 1998c). The potential structures appear to be Gaussian; the electric field signatures fit well to the derivative of a Gaussian. The structures are, in total, nearly charge neutral and carry no significant, statistically-averaged, net potential drop. The peak amplitudes $\left(\Phi_{0}\right)$ can be tens of per cent of the parallel electron temperature $\left(T e_{\|}\right)$. The parallel scale size of the structures monotonically increases with the amplitude of the structures.

Observations of solitary structures in space plasmas has sparked several new theoretical investigations. Electron phase space holes were first reported in laboratory experiments and numerical simulations and later analytically described (Saeki et al., 1979: Schamel, 1982; Turikov, 1984). Solitary structures were found to emerge from electron beams (Omura et al., 1994) and through the two-stream instability (Goldman et al., 1998). Once the structures are developed, the static, parallel (to the magnetic field) properties of the developed solitary structures can be well described by one-dimensional BGK analysis (Muschietti et al., 1999a: 1999b). The mid-altitude auroral structures, however, are clearly two- or three-dimensional structures (Ergun et al., 1998c) with a ratio of perpendicular to parallel scale sizes of $O(1)$. The three-dimensional structures have not yet been described theoretically. 


\section{Observations}

The observations in this article are from the FAST satellite electric and magnetic field instrument (Ergun et al., 1999b) and ion and electron electrostatic analyzers (Carlson et al., 1998a,1998b). One of the most important results of the FAST satellite was the uncovering of particle acceleration by magnetic-field-aligned electric fields in the downward current region of the aurora (Carlson et al., 1998c). The solitary structures that we examine are mostly this region at altitudes from $\sim 2000 \mathrm{~km}$ to $\sim 4000 \mathrm{~km}$. Although solitary structures are more common in the downward current region of the aurora, they also have been observed in the upward current region in association with field-aligned electron beams and have similar characteristics.

\subsection{General observations}

Figure 1 displays a small portion $(25 \mathrm{~s})$ of a pre-midnight auroral crossing in the Northern hemisphere in the downward current region of the aurora as evidenced by the upgoing, energetic, field-aligned electron fluxes (Figs. 1e,f, $180^{\circ}$ pitch angle indicates up-going). The weak, downgoing, field-aligned electron fluxes (Fig. 1f, at $0^{\circ}$ and $360^{\circ}$ ) were the tail of the up-going distribution. The ions fluxes were nearly perpendicular (near $90^{\circ}$ and $270^{\circ}$ pitch angles), indicative of ion heating (Figs. 1g,h). Two bursts of broadband, $\sim 100 \mathrm{~Hz}$ to $\sim 10 \mathrm{kHz}$, electric field emissions (Figs. $1 \mathrm{c}, \mathrm{d}$, marked on the plot) occurred at $-14: 26: 11 \mathrm{UT}$ and at $\sim$ 14:26:19 UT. During these two bursts, ion energies increased (Fig. $1 \mathrm{~g}$ ), ion pitch angles became very close to perpendicular (Fig. 1h), and the current to the Langmuir probe decreased indicating a possible density decrease (Fig. 1a). There also were several visible electric field structures (Fig. 1b).

The enhanced broadband emissions appeared in both the perpendicular and parallel components of the electric field. The parallel electric field (Fig. 1c) had enhanced broadband emissions above $1 \mathrm{kHz}$. The perpendicular electric field (Fig. 1d) had similar enhanced broadband emissions but also had stronger lower-frequency $(\sim 200 \mathrm{~Hz}-\sim 1 \mathrm{kHz})$ emissions that exhibited depletions in power or "bite-outs" at the $\mathrm{H}+$ cyclotron harmonics (Ergun et al., 1998a, Fig. 4). There was a weak perpendicular magnetic component not displayed in the figure. These enhanced emissions are the spectral signature of solitary structures.

Figure 2 gives a closer view $(60 \mathrm{~ms})$ of the electromagnetic fields of the solitary structures from a different orbit than Fig. 1. Panels a-d display the parallel electric field $\left(\Delta E_{\|}\right)$, two components of the electric field $\left(\Delta E_{\perp}\right)$ perpendicular to the ambient magnetic field $\left(B_{0}\right)$, and one component of the perturbation magnetic field $\left(\Delta B_{1}\right)$ perpendicular to $B_{0}$. Panels aa-dd are expanded views $(5 \mathrm{~ms})$ of panels a-d. The parallel electric field signature, $\Delta E_{\|}$, was bipolar, always with the same sense. The first excursion (negative) was in the direction of the energetic electron drift. The second excursion (positive) was opposite the direction of the

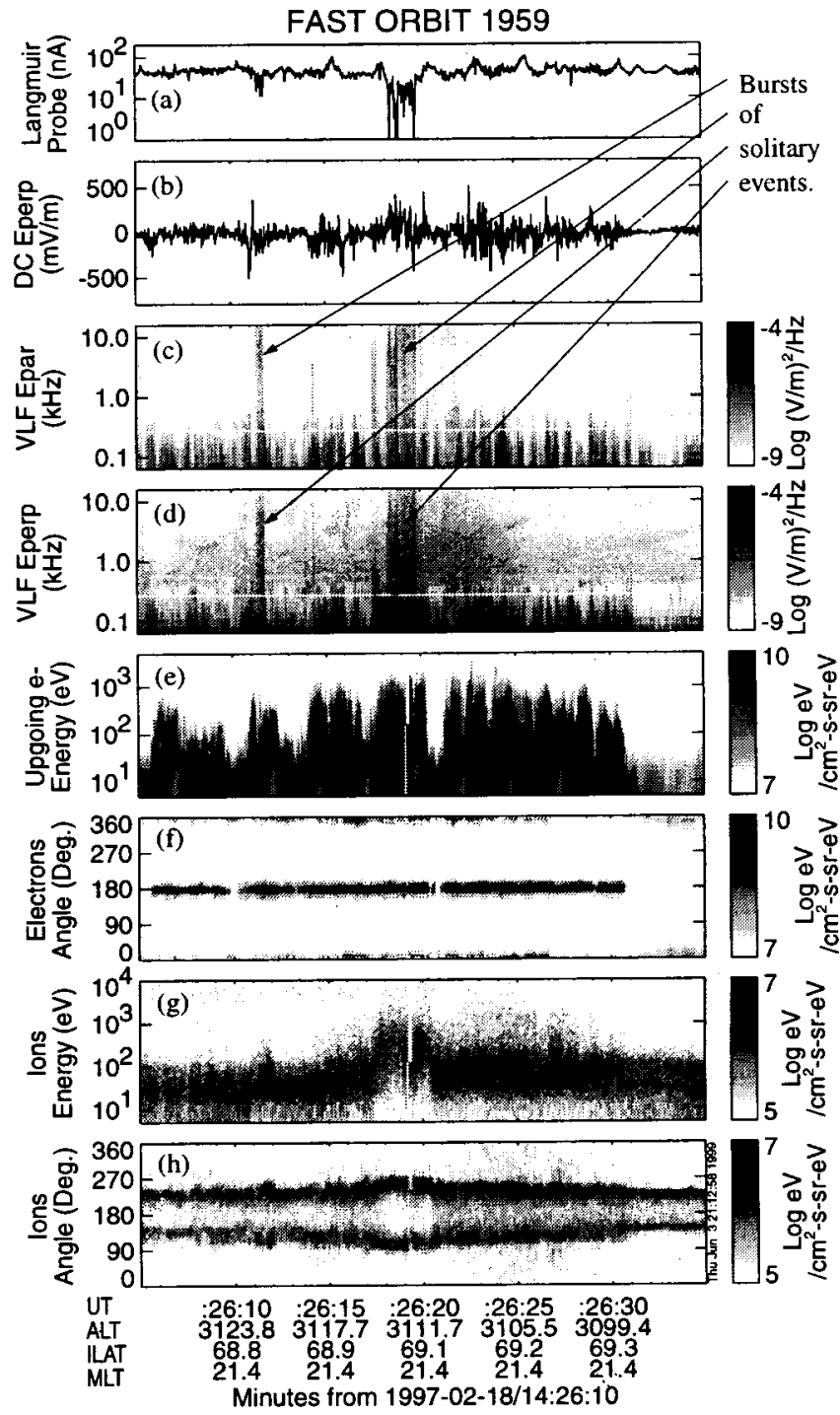

Fig. 1. (From Ergun et al., 1998b) (a) Langmuir probe current reflecting the plasma density. A $2 \mathrm{nA}$ current roughly corresponds to $1 \mathrm{~cm}^{-3}$ density. Fast variations may be capacitively induced current from waves. (b) Perpendicular electric field in the spin plane and nearly along the spacecraft velocity. (c-d) Parallel and perpendicular electric field power spectral density. (e-f) Electron energy flux versus energy and time and versus pitch angle and time. (g-h) Ion energy flux.

electron drift. Both components of $\Delta E_{\perp}$ were unipolar with amplitudes comparable to $\Delta E_{\|}$. The duration of the solitary structures was $\sim 100 \mu \mathrm{s}$. $\Delta B_{\perp}$ was also unipolar and such that $\Delta E_{\perp} / \Delta B_{\perp}>\mathrm{c}$.

The velocity of the structures $\left(v_{s t r}\right)$ can be determined from time delays between physically separated antennas. It also was found that the data were consistent with $\Delta \boldsymbol{E} \cdot \Delta \boldsymbol{B}=0$ and that $\Delta B_{\perp}$ is consistent with the current and displacement current of a moving charge such that $v_{\Delta B} \cong$ $v_{s t r}$ where $v_{\Delta B}=c^{2} \Delta B_{\perp} / \Delta E_{\perp}$. For example, the structure in Figs. 2aa-dd at $\sim 1.2 \mathrm{~ms}$ had the measured component (in the direction of the magnetometer) $\Delta B_{\perp} \cong 6.5 \mathrm{pT}$ while the normal $\Delta E_{\perp} \cong 0.6 \mathrm{~V} / \mathrm{m}$. Thus $\left|v_{\Delta B}\right| \cong 1000 \mathrm{~km} / \mathrm{s}$, which is in 


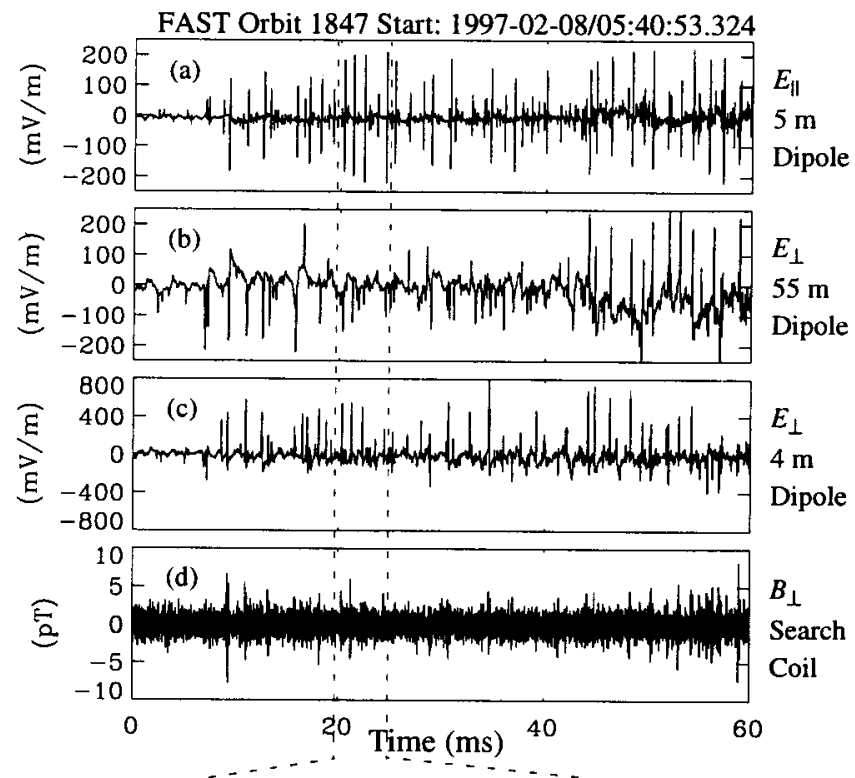

electron drift velocity relative to the ions derived from the first moment of the observed electron distributions:

$$
v_{d}=\int f_{e}(v) v d v^{3} / n
$$

where $f_{e}$ is the electron distribution function and $n$ is the plasma density. In a large $(>1000)$ sample of events, the measured speed $\left(v_{s t r}\right)$ was found to be within a factor of two of the electron drift speed for more that $50 \%$ of the events. The structure velocities are substantially greater that the ion thermal speed.

The observed electromagnetic fields were interpreted to be those of a two- or three-dimensional positively charged cloud (or electron hole) passing by the spacecraft at the electron drift velocity. $\Delta E_{\perp}$ showed no preferred direction, suggesting that the structures are three-dimensional. These charge clouds are accompanied by non-thermal electron distributions. We next look into the detailed electron distributions and electromagnetic properties of solitary structures.

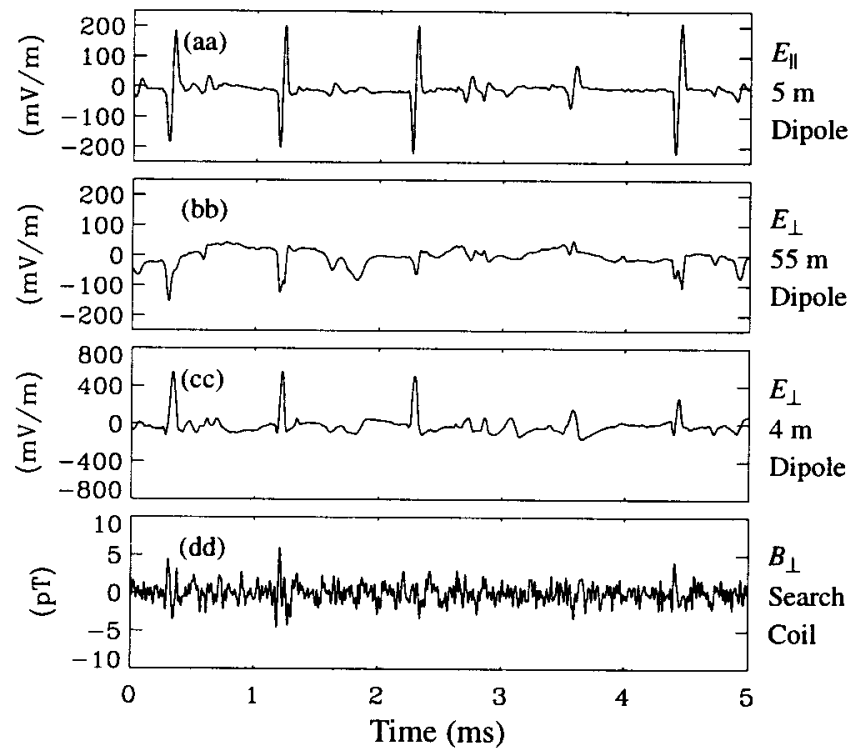

\subsection{Particle distributions}

An example of an electron distribution during solitary wave events is plotted in Fig. 3. It had energetic, field-aligned electrons $\left(T e_{\|} \gg T e_{\perp}\right)$ and was clearly non-thermal. The electron distribution has a drift relative to the spacecraft (or ion) frame. The time-averaged electron distribution anti-parallel to $\boldsymbol{B}_{\mathrm{o}}$ displays a broad plateau extending to $\sim 50 \mathrm{eV}$. In this example, the anti-parallel (to $\boldsymbol{B}_{\mathrm{o}}$ ) distribution dominates over all other pitch-angles producing a substantial drift velocity and current.

The electron distributions best fit a "flat top" with powerlaw tails (Carlson et al., 1998c; Muschietti et al., 1999a) as displayed in Fig. 3. The drift velocity, $v_{d}$, in this example is

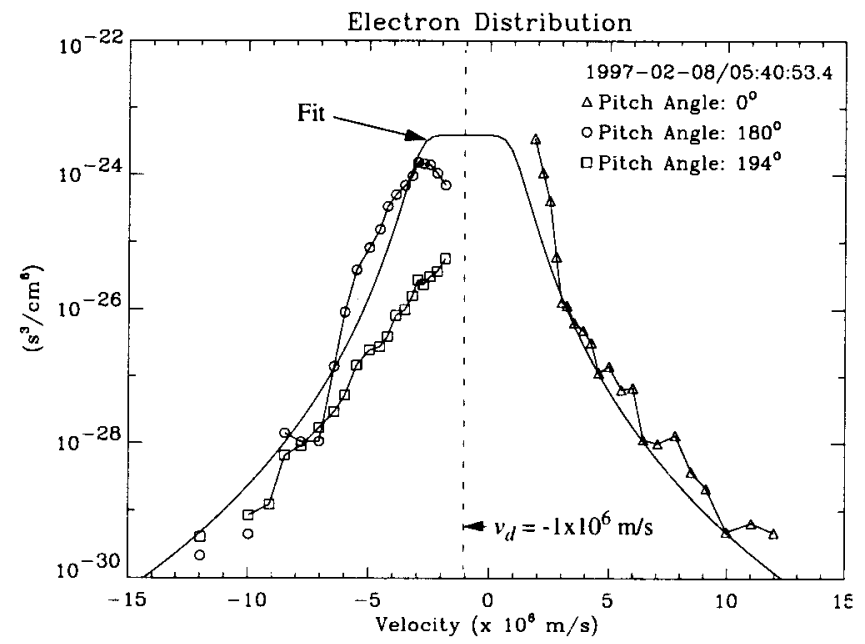

Fig. 2. (From Ergun et al., 1998c) (a) The electric field parallel to $\boldsymbol{B}_{0}$. (b) The electric field perpendicular to $B_{0}\left(\Delta E_{\perp}\right)$ and in the spin plane of the satellite. This signal, measured by a $56 \mathrm{~m}$ dipole antenna, appears attenuated, indicating that the structure size may have been $<112 \mathrm{~m}$. (c) $\Delta E_{\perp}$ along the spin axis of the satellite. (d) A perturbation magnetic field perpendicular to $B_{0}\left(\Delta B_{\perp}\right) . \Delta B_{\perp}$ was filtered to a pass band ( $3 \mathrm{kHz}-16 \mathrm{kHz}$ ) to expose the weak signals and therefore may not appear unipolar in this figure. (aa) (dd) An expanded view of the above data.

agreement with the velocity derived from the time delay ( $v_{s t r}$ is not shown). The solitary structures, therefore, are electrostatic in their rest frame so $\Delta B_{\perp}$ can be used to determine velocity when measured time delays are not available (a specific antenna orientation is needed to measure the time delay). The measured speeds almost always lie between 500 $\mathrm{km} / \mathrm{s}$ and $5000 \mathrm{~km} / \mathrm{s}$. The structures travel along $\boldsymbol{B}_{\mathrm{o}}$ in the same direction and with roughly the same speed as the energetic electrons. The structure velocity corresponds to the

Fig. 3. An electron distribution at three angles from $\boldsymbol{B}_{0}$. The anti-parallel distribution $\left(180^{\circ}\right)$ dominates over all other angles. The distribution at $194^{\circ}$ does not show an enhancement, indicating the electron fluxes were nearly field-aligned. This distribution had $v_{d} \sim 1 \times 10^{6} \mathrm{~m} / \mathrm{s}$ and a $T e_{\|} \sim 30 \mathrm{eV}\left(v_{t h} \sim\right.$ $3 \times 10^{6} \mathrm{~m} / \mathrm{s}$ ). Measurements below $\sim 1 \times 10^{6} \mathrm{~m} / \mathrm{s}$ could be affected by spacecraft charging. 
$\sim-1000 \mathrm{~km} / \mathrm{s}$ while the thermal velocity, defined as the second moment of the distribution in the electron drift frame, was $\sim 3000 \mathrm{~km} / \mathrm{s}$. The ratio of $\left|v_{d} / v_{t h}\right| \sim 1 / 3$ is not uncommon. The occurrence of a small sample of the ratio $\left|v_{d} / v_{t h}\right|$ during solitary waves is displayed in Fig. 4. There is a clear peak at $\left|v_{d} / v_{t h}\right| \sim 0.1$ with only one event with $\left|v_{d} / v_{t h}\right| \sim 1$. This evidence indicates that the electron distributions are rapidly stabilized after being accelerated.

The above electron distributions were compiled on time scales of $\sim 70 \mathrm{~ms}$, far longer than the typical duration of a solitary structure $(100 \mu \mathrm{s})$ and therefore are more representative of the ambient electron distribution rather than that of the charge cloud. On millisecond time scales, the electron distributions associated with the solitary structures display strong modulations (Carlson et al., 1998c). These modulations are consistent with the "charge cloud" interpretation (Muschietti et al., 1999a).

The ion distributions are dominated by heated ions at nearperpendicular pitch angles (Figs. 1g,h). These heated ions are a significant part, if not the majority, of the total ion density. The distributions also contain a more isotropic population that originates from the plasma sheet. There appears to be no "cold" $(<5 \mathrm{eV})$ thermal core.

\subsection{Parallel size}

Figure 5a, a greatly expanded view of $\Delta E_{\mid i}$, has the time axis translated into Debye lengths $\left(\lambda_{\mathrm{D}}\right)$ assuming a constant parallel velocity. The displayed structure had a small $\Delta E_{\perp}$ which indicates that it was either centered about the spacecraft as it passed by or had a large perpendicular scale size. The measured signal (circles) fit remarkably well to a derivative of a Gaussian):

$$
E(z)=\frac{E_{o} x}{x_{o}} e^{-\frac{1}{2}\left(x / x_{0}\right)^{2}}
$$

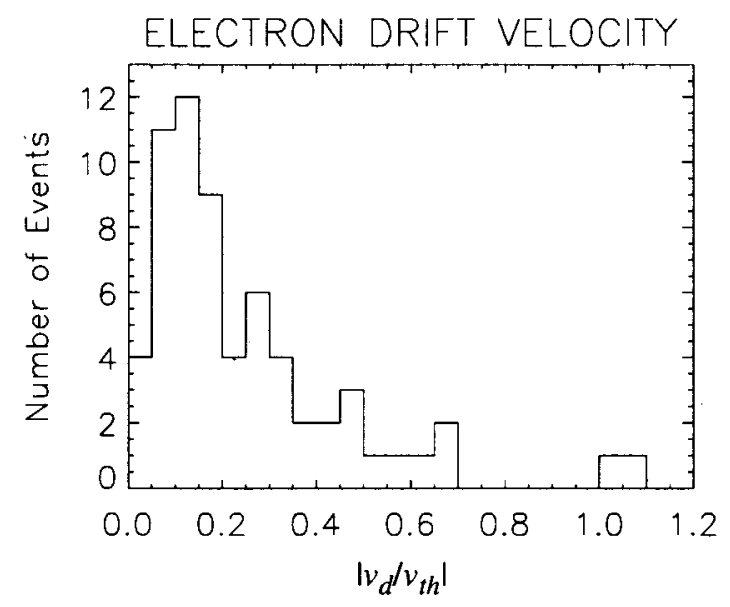

Fig. 4. A histogram of a small sample of the ratio of the electron drift velocity to the electron thermal velocity (second moment of the electron distribution in the drift frame) when solitary structures were observed. The ratio $\left|v_{d} / v_{t h}\right|$ peaks $<0.2$ indicting that wave-particle interactions rapidly thermalize the accelerated electrons.
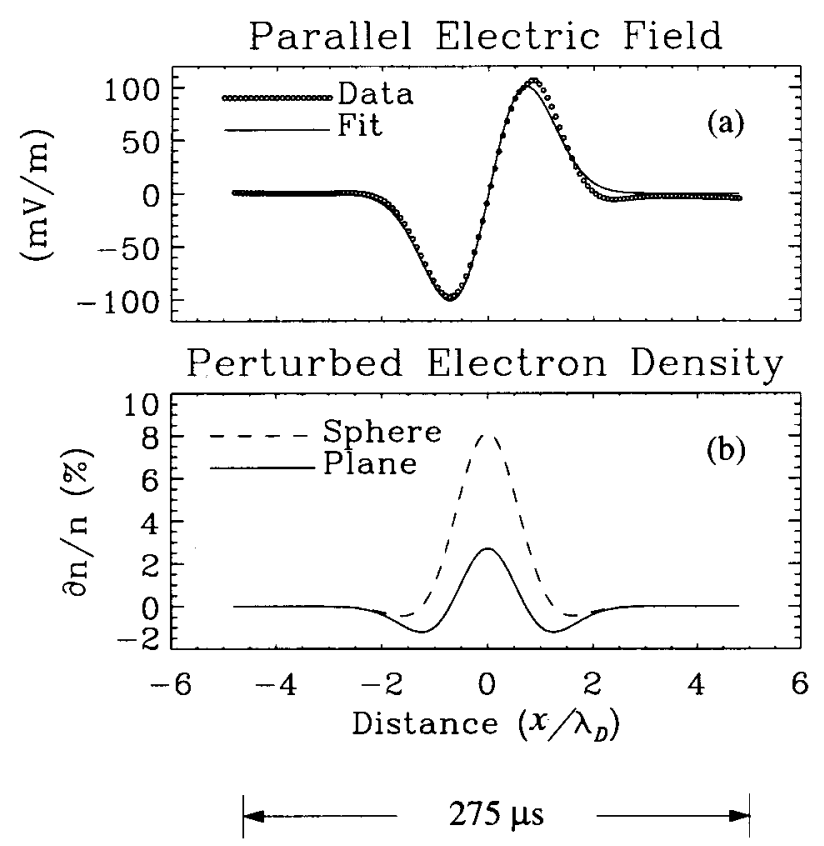

Fig. 5. (From Ergun et al., 1998c) (a) $\Delta E_{\|}$. The dots are the data at $0.5 \mu \mathrm{s}$ resolution translated into Debye lengths assuming a constant parallel velocity, $v_{s o l}=3.2 \times 10^{6}$. The smooth trace is the fit to Eq. (2). (b) Calculated charge densities assuming spherical and planar geometry. The plasma conditions were $n_{o}=5.7 \pm 2.0 \mathrm{~cm}^{-3}, T_{e_{||}}=704 \pm 145 \mathrm{eV}, v_{\text {sol }}=$ $3.2 \times 10^{6} \pm 1.1 \times 10^{6} \mathrm{~m} / \mathrm{s}, T_{\perp}=370 \pm 74 \mathrm{eV},\left|B_{\mathrm{o}}\right|=11481 \pm 10 \mathrm{nT}, \lambda_{\mathrm{D}}=82 \pm$ $30 \mathrm{~m}$, and $\rho_{\mathrm{H}+}=241 \pm 24 \mathrm{~m}$.

The solid line in Fig. 5a is a fit to the data (circles) with $x_{o}=$ $0.7 \pm 0.3 \lambda_{\mathrm{D}}$. The local plasma conditions are summarized in the figure caption.

The charge density of a Gaussian structure is well known. The actual charge density of the observed structure lay between the two traces in Fig. $5 \mathrm{~b}$ which represent spherical and planar geometry. The structures (which are nearly spherical, see below) have a positive core such that the deviation from charge neutrality is roughly $8 \%$ (in the above example which is fairly representative) of the ambient plasma density $\left(n_{o}\right)$. The positive core is surrounded by a negative halo. A close examination of $\Delta E_{\|}$(Fig. 5a) reveals that it abruptly begins and ends which implies that the structures are, in total, charge neutral.

We examined over one thousand events to determine the statistical properties of fast solitary structures. The solitary structures were chosen by an algorithm that was optimized to ensure the selected events were solitary structures (as one would identify by eye) rather than to locate all events. The primary selection criteria isolated bipolar (asymmetry less than a factor of two), parallel electric field signals with peaks exceeding the surrounding RMS amplitude, averaged over $\sim 2 \mathrm{~ms}$, by a factor of five. The perpendicular electric field had to be nearly unipolar, that is, have an asymmetry of greater than a factor of two. Events with amplitudes larger than $200 \mathrm{mV} / \mathrm{m}$ were not included in some parts of the study because of possible sensor saturation. A spot check indicated that over one half of the structures discernible by eye 
were not identified, especially those with low $(<50 \mathrm{mV} / \mathrm{m})$ amplitudes.

The average size (Gaussian half-width, $x_{o}$ ) of the structures parallel to $B_{\mathrm{o}}$ was found to be $1.80 \lambda_{\mathrm{D}}$ with a standard deviation of $1.13 \lambda_{\mathrm{D}}$. The scale size was estimated as $\Delta t_{p p} v_{s t r} / 2$ where $\Delta t_{p p}$ is the time between the positive peak and negative peak and $v_{s t r}$ is the velocity of the solitary structure. The Debye length, $\lambda_{D}$, was determined from the measured electron temperature and the ion density (the ion density measurement had the best accuracy). In most cases, $\lambda_{\mathrm{D}}$ and $\Delta_{p p}$ were determined to better than $25 \%$. The velocity, $v_{s t r}$ however, could not be as accurately determined. It often could only be determined to within a factor of two. The measured delay between two sensors was used if the antennae were favorably oriented. The Lorentz velocity $\left(v_{\Delta B}\right)$ was used if $\Delta B_{\perp}$ was detectable. The standard deviation was influenced by the uncertainty in $v_{\text {str. }}$

The average peak potential $\left.\left(<\Phi_{0}\right\rangle\right)$ was examined with different selection criteria. Events with amplitudes up to 1 $\mathrm{V} / \mathrm{m}$ were included. The results of the study are plotted in Fig. 6. $\Phi_{0}$ was found to be most often $<0.2$ of the parallel electron temperature $\left(T e_{\|}\right)$. Curiously, the occurrence of $\Phi_{\mathrm{o}}$ ' $T e_{\|}$of the structures seemed to obey a power law of $\sim-1.5 \pm$ 0.3 suggesting a turbulence scaling law. Note that the occurrence of $\Phi_{0} / T e_{\|}<0.1$ was influenced by the selection criteria so one cannot determine an average or median potential. There were very few events with $\Phi_{0} / T e_{\|}>1$.

Finally, a statistical study was carried out to determine if the solitary structures carry a net potential drop across the structure $(\Delta \Phi)$. The selection criteria limited the study to events that had an asymmetry in the parallel signal no greater than a factor of two. These structures, on average, had no statistically significant net potential $\left(\left\langle\Delta \Phi / \Phi_{0}\right\rangle \sim 0\right)$ and a standard deviation of $\Delta \Phi / \Phi_{0} \sim 5 \%$. The apparently random asymmetry could come from a number of factors including background plasma waves, growth or decay of the structure, or random net potentials. This evidence suggests

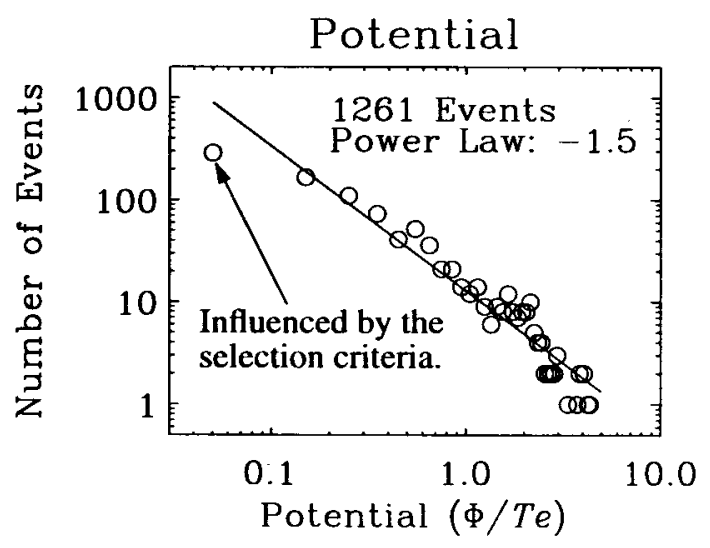

Fig. 6. A histogram of the amplitude $\left(\Phi_{0}\right)$ of solitary structures relative to the electron temperature $\left(T e_{\|}\right)$. The selection algorithm did not properly identify small-amplitude events, so the number of events s with $\Phi_{t} / T e_{|| \mid}<$ 0.1 is not correct. The occurrence appears to follow a power law. that the solitary structures do not directly support a parallel electric field.

\subsection{Parallel size-amplitude relation}

The relationship between the amplitude and the size of the solitary structures gives us some insight on the nature of the structures. Under a simple self-focusing process, for example the Korteweg-deVries solution, the amplitude should increase with decreasing size of the solitary structure whereas the one-dimensional "electron phase space hole" solution (Turikov et al., 1984) predicts the opposite.

The relationship between $\Phi_{0}$ and size $\left(x_{0}\right)$ is displayed in Fig. 7. $\Phi_{0}$ clearly increases with size. The general shape of the curve, when $x_{o} / \lambda_{\mathrm{D}}<2$, agrees with analytical results and particle simulations of one-dimensional electron phase space holes (Turikov et al., 1984; Muschietti et al., 1999a; $1999 \mathrm{~b}$ ). The observed structures are predicted to be stable in one-dimension if their velocity is less than twice the electron thermal speed and they are moving at many times the ion sound speed. The observed structures satisfy that criteria.

The structure's properties parallel to $B_{0}$ can be understood as a pure electron phenomena (BGK modes) in one dimension (Muschietti et al., 1999a). The one-dimensional assumption has validity because the electrons are strongly magnetized $\left(\rho_{e}<<\lambda_{D}\right)$. The event above, for example, has $\rho_{e}<1 \mathrm{~m}$ and $\lambda_{\mathrm{D}} \sim 82 \mathrm{~m}$. The excellent agreement between the measured amplitude-size relationship and that from BGK analysis (Fig. 7) indicates that the observed structures are in a quasi-stable saturated state.

\subsection{Perpendicular size}

Theoretically, one expects ions to control the perpendicular scale size. The electrons are strongly magnetized $\left(\rho_{e} \ll\right.$ $\lambda_{\mathrm{D}}$ ), restricting their motion one dimension. Observational

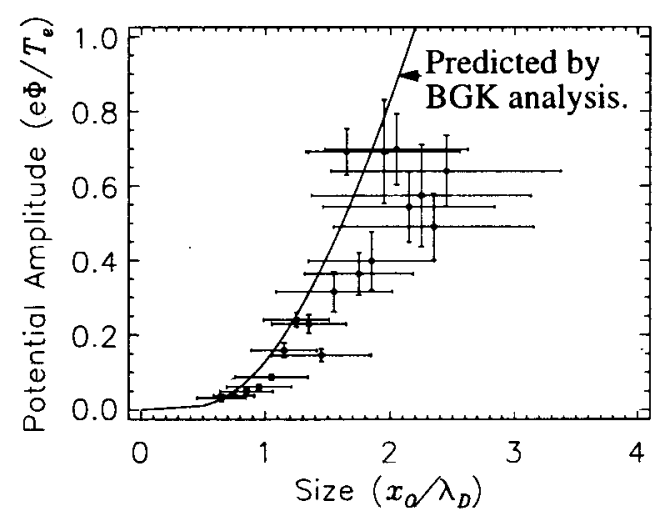

Fig. 7. The peak potential $\left(\Phi_{0} / T e_{\|}\right)$versus the structure size $\left(x_{0} / \lambda_{\mathrm{D}}\right)$. Each point reflects the mean potential and size averaged over a size bin. The solid line is the minimum size predicted by BKG analysis (Muschietti et al., 1999b). 
evidence also suggests that ion dynamics has a role. Occasionally the structures are periodically spaced close to the $\mathrm{H}+$ cyclotron frequency (Ergun et al., 1998c) and the spectral power density of the electric field waveforms often shows structure at the $\mathrm{H}+$ cyclotron harmonics (Ergun et al., 1998a). Solitary structures also have been observed in sets where the spatial extent perpendicular to the magnetic field was on the order of the $\mathrm{H}+$ Larmor radii. The observations suggest that the perpendicular dimension scales with the ion gyro-radius $\left(\rho_{\mathrm{i}}\right)$ and that solitary structures have a source region confined to a flux tube.

In the mid-altitude auroral region, $\Delta E_{\|}$and $\Delta E_{\perp}$ are typically comparable and hodegrams of $\Delta E_{\|}$versus $\Delta E_{\perp}$ often conform to a spheroid such that $x_{o} \leq r_{o}$, where $x_{o}$ is the parallel (to $\boldsymbol{B}_{\mathrm{o}}$ ) scale size and $r_{o}$ the perpendicular scale size. One cannot, however, determine the oblateness of the charge cloud from a single event since it is not possible to determine where in the cloud the observation was made (near the center or the edge). The oblateness must be determined statistically. The selection criteria, however, were biased. Only events with a clear bipolar parallel electric field were chosen. Events at the edge of the charge cloud were ignored. The curve in Fig. 8 accounts for a bias in the selection criteria. With the bias removed, the statistical scale size has $r_{o}$ $1.4 x_{o}$ (Fig. 8). Interestingly, the proton gyro-radius $\left(\rho_{\mathrm{i}}\right)$ was $\sim 2 \lambda_{\mathrm{D}}$ on average.

Auroral solitary waves observed at much higher altitudes appear to be much more oblate $\left(r_{o} \gg x_{o}\right)$ (Franz et al., 1988). In these regions $\rho_{i} \gg \lambda_{D}$. These results suggested

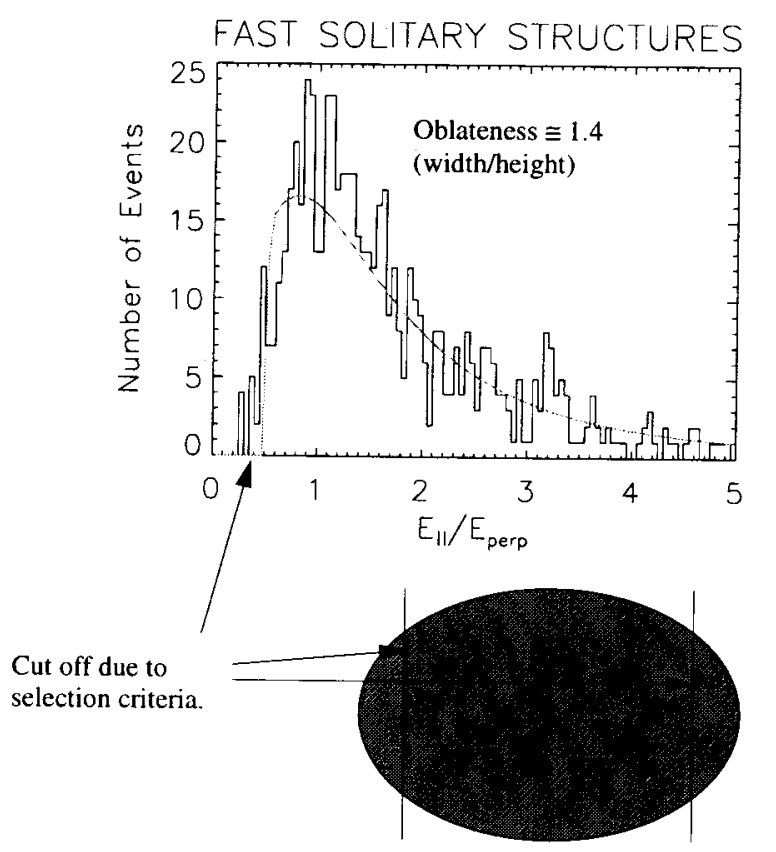

Fig. 8. A histogram of the ratio $\Delta E_{||} / \Delta E_{\perp}$. The sharp cutoff at $\sim 0.5$ is due to the selection eriteria. With the selection criteria properly accounted for, the histogram fits well to that expected from solitary structures with an oblateness of 1.4 . that the oblateness of the spheroids depends upon to the ratio of $\rho_{i}$ and $\lambda_{D}$. Thus, observational evidence suggests that the parallel size is controlled by $\lambda_{\mathrm{D}}$ and the perpendicular size is controlled by $\rho_{i}$.

\subsection{Ion heating}

Ion distributions show a clear signature of enhanced heating when solitary structures are present (Figs. $1 \mathrm{~g}, \mathrm{~h}$ ). Ion heating results from multi-dimensional structures. In the frame of the solitary structure, ions are incident at very high velocity compared to their thermal velocity, and will scatter from the positively charged core. The scattering angle is small. We provide a simple estimate. Assuming the perturbation to the ion trajectory is small and that the ion are unmagnetized (in other words, the ion transit time is far less than the gyroperiod), the perpendicular impulse from an proton passing through the solitary structure with an impact parameter of $r$ is:

$$
M \Delta v_{i \perp}(r)=\int_{-\infty}^{\infty} e E_{\perp}(r, z(t)) d t
$$

where $M$ is the proton mass.

In the ion frame, the particles experience many such perpendicular impulses. If the impulses are random in time or direction, the ions heat stochastically. Figure 9 demonstrates the heating of an individual proton and the average over 1000 particles under a impulse rate of 1000 structures per second with a perpendicular electric field amplitude of 140

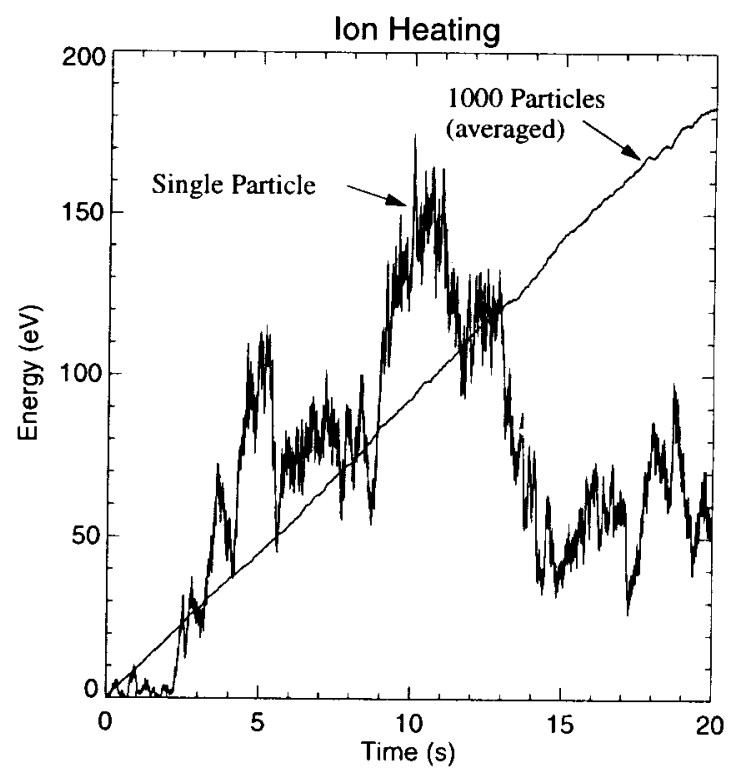

Fig. 9. Protons gyrating in a uniform $10000 \mathrm{nT}$ magnetic field experience a $1.4 \times 10^{-5} \mathrm{~V}-\mathrm{s} / \mathrm{m}$ perpendicular electric field impulse at a rate of 1000 impulses per second (randomly directed). The impulse is that of a $140 \mathrm{mV}$ / $m$ electric field with a duration of $100 \mu \mathrm{s}$. The smooth trace is the average heating rate of 1000 particles. The noisy trace follows an individual particle. The average heating rate is $-10 \mathrm{eV} / \mathrm{s}$, which can account for the observed enhancement of ion heating. In extreme cases, the heating rate can exceed $100 \mathrm{eV} / \mathrm{s}$ leading to kilovolt ion energies. 
$\mathrm{mV} / \mathrm{m}$ and duration of $100 \mu \mathrm{s}$. The simulation starts with a proton at rest in a uniform magnetic field of $10^{-5} \mathrm{~T}$. The perpendicular electric field is treated as an abrupt impulse $\left(1.4 \times 10^{-5} \mathrm{~V}-\mathrm{s} / \mathrm{m}\right)$ in a random direction. There were 1000 impulses a second. The ion energy is recorded after each impulse. The simulation was repeated 1000 times to determine an average heating rate. The average heating rate of $\sim 10 \mathrm{eV} / \mathrm{s}$ can account for the observed enhancement of ion heating. Since the heating is proportional to $\Delta E_{\perp}{ }^{2}$, the heating rate can exceed $100 \mathrm{eV} / \mathrm{s}$ in extreme cases leading to kilovolt ion energies.

\section{Summary}

Solitary structures have been observed in several regions of the magnetosphere and may be an indicator of local electron acceleration. The FAST satellite observations in the midaltitude aurora are among the most detailed to date. From these observations we have compiled the following list of properties.

\subsection{Geophysical properties}

1. Fast solitary structures are common in the downward current region that was surveyed by FAST. They are only seen in regions of energetic, field-aligned electron fluxes.

2. Fast solitary structures are observed in upward current region less frequently than in the downward current region, and only with field-aligned electron fluxes.

3. Fast solitary structures are observed on magnetic field lines that have parallel electric fields.

4. Fast solitary structures are associated with enhanced ion heating. Ion pitch angles are found near $90^{\circ}$ during intense emissions.

\subsection{Physical properties}

5. The $E_{\|}$spectra have enhanced, broad band power at 1 $10 \mathrm{kHz}$. The $E_{\perp}$ spectra have similar enhanced, broad band power and absorption at the ion cyclotron harmonics.

6. The typical duration is $\sim 50 \mu$ s to $\sim 200 \mu \mathrm{s}$, with a range of $\sim 20 \mu \mathrm{s}$ to $\sim 500 \mu \mathrm{s}$ (the ion cyclotron period).

7. $\Delta E_{\|}$is bipolar. Amplitudes often are above $50 \mathrm{mV} / \mathrm{m}$ and reach as high as several $\mathrm{V} / \mathrm{m}$.

8. The parallel profile fits well to a derivative of a Gaussian.

9. The parallel scale size (Gaussian half-width) is roughly $2 \lambda_{\mathrm{D}}$ (typically $\sim 100 \mathrm{~m}$ ).

10. The maximum potential ( $e \Phi_{\mathrm{o}}$ ) of the structures can be tens of per cent of $T e_{\|}$.

11. Solitary structures carry no (statistical) average net potential drop $\left.\left(<\Delta \Phi / \Phi_{0}\right\rangle \sim 0\right)$.

12. $\Delta E_{\perp}$ is unipolar. $\Delta E_{\|}$and $\Delta E_{\perp}$ are typically comparable.

13. The statistically determined oblateness $\left(r_{0} / x_{0}\right)$ in the mid-altitude $(-2000 \mathrm{~km}$ to $\sim 4000 \mathrm{~km})$ auroral region is $\sim 1.4$.

14. The structures are occasionally organized near the $\mathrm{H}+$ cyclotron or lower hybrid frequency.

15. $\Delta B$ is unipolar, perpendicular, and such that $\left|\Delta E_{\perp}\right| /|\Delta B|$ $\sim 100 c$ and $\Delta \boldsymbol{E} \cdot \Delta \boldsymbol{B}=0$.

16. The ratio $|\Delta E| /|\Delta B|$ is consistent with $c^{2} / v_{\text {str }}$

17. The speed of the structures is typically between $500 \mathrm{~km} /$ $\mathrm{s}$ and $5000 \mathrm{~km} / \mathrm{s}$.

18. Solitary structures have speeds roughly that of the electron drift velocity.

19. The attendant electron distributions are drifting "flattop" distributions with $\left|v_{d} / v_{t h}\right| \sim 0.2$.

20. The attendant electron distributions are strongly fieldaligned.

21. Strong electron modulations are associated with these structures.

22. The attendant ion distributions are roughly $90^{\circ}$ conics which have enhanced perpendicular heating over the surrounding regions.

23. The near-perpendicular heated ions dominate the ion density.

24. Large-amplitude solitary structures can energize ions at $>10 \mathrm{eV} / \mathrm{s}$.

25. Observational evidence suggests that the parallel size is controlled by $\lambda_{D}$ and the perpendicular size is controlled by $\rho_{\mathrm{i}}$.

Acknowledgments. The authors thank M. V. Goldman and D. L. Newman for constructive discussions. This work was supported by NASA grants NAG5-3596 and NAG5-6985.

\section{References}

Bale, S. D., Kellogg, P. J., Larson, D. E., Lin, R. P., Goetz, K., and Lepping, R. P., Bipolar electrostatic structures in the shock transition region: Evidence of electron phase space holes, Geophys. Res. Lett., 25, 2929, 1998.

Carlson, C. W., Pfaff, R., and Watzin, J. G., The Fast Auroral SnapshoT (FAST) mission, Geophys. Res. Lett., 25, 2013, 1998a.

Carlson, C. W. and McFadden, J. P., Design and applications of imaging plasma instruments, in Monog. Meas. Techn. Space Plasma, edited by R, Pfaff, AGU, 1998b.

Carlson, C. W., McFadden, J. P., Ergun, R. E., Temerin, M., Peria, W., Mozer, F. S., Klumpar, D. M., Shelly, E. G., Peterson, W. K., Moebius, E., Elphic, R., Strangeway, R. J., Cattell, C., and Pfaff, R., FAST observations in the downward auroral current region: Energetic upgoing electrons beams, parallel potential drops, and ion heating, Geophys. Res. Lett., 25 , 2017, 1998c.

Dubouloz, N., Pottelette, R., and Malingre, M., Generation of broadband electrostatic noise by electron acoustic solitons, Geophys. Res. Lett., 18, $155,1991$.

Ergun, R. E., Carlson, C. W., McFadden, J. P., Mozer, F. S., Delory, G. T., Peria, W., Chaston, C. C., Temerin, M., Elphic, R., Strangeway, R. J., Pfaff, R., Cattell, C. A., Klumpar, D., Shelly, E., Peterson, W., Moebius, E., and Kistler. L., FAST satellite observations of electric field structures in the auroral zone, in press, Geophys. Res. Lett., 25, 2025, 1998a.

Ergun, R. E., Carlson, C. W., McFadden, J. P., Mozer, F. S., Delory, G. T., Peria. W., Chaston, C. C., Temerin, M., Elphic, R., Strangeway, R. J., Pfaff, R., Cattell. C. A., Klumpar, D., Shelly. E., Peterson. W., Moebius, E., and Kistler, L., FAST satellite observations of large-amplitude solitary structures, Geophys. Res. Lett., 25, 2041, 1998 b. 
Ergun, R. E., Carlson, C. W., McFadden, J. P., Mozer, F. S., Muschietti, L., and Roth, I., Debye-scale plasma structures associated with magneticfield-aligned electric fields, Phys. Rev. Lett., 81, 826, $1998 \mathrm{c}$.

Ergun, R. E., Magnetic-field-aligned electric fields associated with Debyescale plasma structures, Plasma Phys. Control. Fusion, 41, A61, 1999a.

Ergun, R. E., Carlson, C. W., Mozer, F. S., Delory, G. T., McFadden, J. P., Temerin, M., Pankow, D., Abiad, R., Harvey, P., Primbsch, H., Wilkes, R., Elphic, R., Strangeway, R., Pfaff, R., and Cattell, C. A., The FAST satellite electric and magnetic field instrument, submitted, Space Sci. Rev., 1999b.

Franz, J. R., Kintner, P. M., and Pickett, J. S., Polar observations of coherent electric field structures, Geophys. Res. Lett, 25, 1277, 1998.

Goldman, M. V., Oppenheim, M. M. and Newman, D. L. PIC simulations of bipolar wave structures driven by dense beams in the auroral ionosphere, in Physics of Space Plasmas 15, edited by T. Chang and J. R. Jasperse, p. 115, Cambridge, MA, 1998.

Matsumoto, H., Kojima, H., Miyatake, T., Omura, Y., Okada, M., Nagano, I., and Tsutsui, M., Electrostatic solitary waves (ESW) in the magnetotail: BEN wave forms observed by GEOTAIL, Geophys. Res. Lett., 21, 2915, 1994.

Mozer, F. S., Ergun, R. E., Temerin, M., Cattell, C., Dombeck, J., and Wyg- ant, J., New features of time domain electric-field structures in the auroral acceleration region, Phys. Rev. Lett., 79, 1281, 1997.

Muschietti, L., Ergun, R. E., Roth, I., and Carlson, C. W., Phase-space electron holes along magnetic field lines, Geophys. Res. Lett., 26, 1093, 1999a.

Muschietti, L., Ergun, R. E., Roth, I., and Carlson, C. W., Correction to "Phase-space electron holes along magnetic field lines", Geophys. Res. Lett., 26, 1689, 1999 b.

Omura, Y., Kojima, H., and Matsumoto, H., Computer simulation of electrostatic solitary waves: a nonlinear model of broadband electrostatic noise, Geophys. Res. Lett., 21, 2923, 1994.

Saeki, K., Michelsen, P., Pecseli, H. L., and Rasmussen, J. J., Formation and Coalescence of Electron Solitary Holes, Phys. Rev. Lett., 42, 501, 1979.

Schamel, H., Stability of electron vortex structures in phase space, Phys. Rev. Lett., 48, 481, 1982.

Temerin, M., Cerny, K., Lotko, W., and Mozer, F. S., Observations of double layers and solitary waves in the auroral plasma, Phys. Rev. Lett., 48, 1175 , 1982.

Turikov, V. A. Electron phase-space holes as localized BGK solutions, Physica Scripta, 30, 73, 1984. 